\title{
Rare Complications of Kikuchi's Disease: Beyond Pain Control
}

Mechanicsville, VA, USA

Shailendra Kapoor, MD

We read the recent article by Park et al. [1]. with great interest. Intriguingly, many rare complications may occur as a result of Kikuchi-Fujimoto disease (KFD).

Peripheral neuropathy is one such rare complication of KFD [2]. Limb paresis secondary to involvement of the brachial plexus was reported in a 22-year-old female with KFD [3]. Similarly, KFD may rarely affect the eye, resulting in panuveitis [4]. The disease may also cause ocular vasculitis and subretinal macular infiltrates. Patients with panuveitis may require methotrexate therapy.

In addition, rare complications involving the CNS may occur. For instance, meningitis has been reported in some cases [5]. Aseptic encephalitis has also been occasionally reported. Other patients have presented with cerebellar ataxia.

Cardiac complications such as tamponade may also rarely occur. KFD may additionally result in axillary lymphadenopathy [6]. Isolated mediastinal lymphadenopathy has also been reported [7], and the mesenteric lymph nodes may rarely be involved [8].

KFD may also result in acute renal failure [9]. Hepatitis is another rare complication. In addition, KFD may appear as SLE [10]. Hemophagocytic syndrome has also been reported as a complication of the disease [11]. The lungs may additionally be affected in rare cases, resulting in inter- stitial lung disease. The pleura may also be affected, leading to pleural effusion [12].

Rarely, KFD may occur following Hodgkin's lymphoma [13]. Similarly, Still's disease may be complicated by the appearance of KFD [14]. KFD may also infrequently occur following immunizations. For instance, the Japanese encephalitis virus vaccine, as well as the HPV vaccine, have been implicated as possible triggers of KFD [15]. KFD may also occasionally follow stem cell transplantation [16].

The above examples clearly detail the rare complications of KFD. Physicians should consider KFD in the differential diagnosis of patients presenting with these manifestations.

\section{REFERENCES}

1. Park KE, Kang S, OK SH, Shin IW, Sohn JT, Chung YK, et al. A patient with Kikuchi's disease: what should pain clinicians do? Korean J Pain 2012; 25: 188-90.

2. Longaretti P, Savasta S, Caimmi D, Possenti I, Marseglia GL. Kikuchi-Fujimoto disease complicated by peripheral neuropathy. Pediatr Neurol 2012; 46: 319-21.

3. Pérez Alvarez MJ, Moreno López M. Panuveitis as a possible ophthalmic complication of Kikuchi-Fujimoto disease. Arch Soc Esp Oftalmol 2005; 80: 41-4.

4. Sugiyama A, Araki E, Arakawa K, Kikuchi H, Iwaki T, Yamada

Received August 16, 2012. Accepted August 20, 2012.

Correspondence to: Shailendra Kapoor, MD

74 Crossing, Mechanicsville, VA-23111, USA

Tel: +1-8675675678, Fax: +1-8656071014, E-mail: shailendrakapoor@yahoo.com

() This is an open-access article distributed under the terms of the Creative Commons Attribution Non-Commercial License (http:// creativecommons.org/licenses/by-nc/3.0/), which permits unrestricted non-commercial use, distribution, and reproduction in any medium, provided the original work is properly cited.

Copyright (c) The Korean Pain Society, 2012 
T, et al. A case of subacute necrotizing lymphadenitis complicated with brachial plexus neuritis. Rinsho Shinkeigaku 1998; 38: 941-4.

5. Toledano Muñoz A, García de Casasola G, Argüelles Pintos M, de los Santos Granados G. Kikuchi-Fujimoto disease: report of two cases. Acta Otorrinolaringol Esp 2006; 57: $152-4$.

6. Diez-Morrondo C, Pantoja-Zarza L, Manjón-Haces JA. Kikuchi Fujimoto's disease appearing as systemic lupus erythematosus. Reumatol Clin 2012; 8: 153-5.

7. Verroiotou M, Mogrampi SA, Polytidis I, Fardellas I. Kikuchifujimoto disease: a rare case of axillary lymphadenopathy. Lymphat Res Biol 2011; 9: 115-6.

8. Yoshida Y, Matsuzawa Y, Rikitake H, Wakabayashi T, Okada $N$, Kuroda $T$, et al. Mediastinal lymphadenopathy without cervical lymphadenopathy in a case of Kikuchi-Fujimoto disease. Intern Med 2011; 50: 649-52.

9. Vijayaraghavan R, Chandrashekar R, Saraswathi A, Belagavi CS. Kikuchi-Fujimoto's disease involving mesenteric nodes: a report and review of literature. BMJ Case Rep 2011; 2011. pii: bcr1020114945.

10. Silva AF, Focaccia R, Oliveira AC, Sementilli A, Reis GF. Kikuchi-Fujimoto disease: an unusual association with acute renal failure. Braz J Infect Dis 2010; 14: 621-7.
11. Kim HA, Im SA, Chung NG, Kang JH, Park GS. Disseminated Kikuchi disease associated with hemophagocytic syndrome in an infant: whole-body MRI. Indian J Pediatr 2011; 78: 616-9.

12. Garcia-Zamalloa A, Taboada-Gomez J, Bernardo-Galán P, Magdalena FM, Zaldumbide-Dueñas L, Ugarte-Maiztegui M. Bilateral pleural effusion and interstitial lung disease as unusual manifestations of Kikuchi-Fujimoto disease: case report and literature review. BMC Pulm Med 2010; 10: 54.

13. Joudeh AA, Al-Abbadi MA, Rahal MM, Amr SS. Kikuchi Fujimoto Disease (histiocytic necrotizing lymphadenitis) following Hodgkin lymphoma. Pathol Int 2012; 62: 571-3.

14. Yamamoto T. Cutaneous manifestations associated with adult-onset Still's disease: important diagnostic values. Rheumatol Int 2012; 32: 2233-7.

15. Watanabe T, Hashidate H, Hirayama Y, linuma Y. KikuchiFujimoto disease following vaccination against human papilloma virus infection and Japanese encephalitis. Eur J Pediatr 2012; 171: 1409-11.

16. Lin CC, Yeh SP, Chiang IP, Chiu CF. Kikuchi-Fujimoto disease after allogeneic hematopoietic SCT mimicking post transplant lymphoproliferative disorder. Bone Marrow Transplant 2011; 46: 1389-90. 